\title{
Design of MEMS Oscillator for High Frequency Application
}

\author{
G.Divya, M.Vinothkumar \\ ECE Department SRM University, Delhi-NCR
}

\begin{abstract}
MEMS Oscillator design for high frequency application is approached in this project and its gain, noise and power are analyzed. The CMOS Micro Electro Mechanical System resonator structure is modeled using its RLC equivalent circuit where all passive components are extracted by COMSOL Mutiphysics tool. MEMS based oscillator requires to be combined with amplifier circuits to improve the performance. The proposed CMOS-MEMS oscillator is capable of generating $19.8 \mathrm{MHz}$ clocks. The beam resonators require approximately $35 \mathrm{VDC}$ and $400 \mathrm{mV}, \mathrm{VAC}$ to vibrate. The operational amplifier achieves a gain of $26 \mathrm{~dB}$ at VDD of $1.8 v$ and VSS of $-1.8 v$. For this project, the MEMS based Oscillator circuit was elected due to its quality and high frequency stability. The Micro Electro Mechanical System beam resonator translates to motional resistance of $R_{m}=2.8 \mathrm{M} \Omega$, capacitance, $C_{m}=2.46 \mathrm{~F}$ and inductance, $L_{m}=13.6 \mathrm{H}$ respectively. The MEMS based Oscillator was simulated and measured using Cadence virtuoso to obtain the desired design parameters.
\end{abstract}

Keywords: MEMS resonator, two stage Op-amp, gain, Cadence virtuoso, COMSOL Multi physics.

\section{Introduction}

Resonators based on MEMS (Micro Electro Mechanical Systems) span a broad spectrum of important current applications, including detection of chemical and biological substances, measurement of rheological properties of fluids, and energy harvesting, to name only a few. While the devices that perform these diverse functions span an equally broad range in geometric layout, material properties, circuitry, fabrication techniques, packaging, and so on, they all have one aspect in common: the phenomenon of "resonance" forms the basis of their operating principle. More specifically, they usually perform their desired functions by monitoring how interactions with the environment influence the resonant behavior of the device. Conversely, how effectively the device performs its function will depend to a large degree on the underlying resonant characteristics of the device .Since all such devices rely on resonant vibrations to accomplish one or more tasks effectively, a firm understanding of this highly interdisciplinary field requires that one be familiar with the fundamental theory of mechanical vibrations. This paper presents a fixed-fixed beam MEMS Resonator. The resonating beam is fixed at both ends, called Anchor that is why called fixed-fixed Resonator. In this paper, fixed-fixed beam resonator is modeled by COMSOL Multi physics software. A lot of modes of vibration can be found in a rectangular plate resonator either by changing the thickness of electrodes or by changing the mode number. MEMS resonators are small electromechanical structures that vibrate at high frequencies. From the industrial perspective, these technologies promise to deliver exactly to their applications needs. The resulting achievable goals are devices in the form of high-capability with more embedded functional features, bearing the attributes of portability and wear-ability. MEMS are widely used for diverse applications in high $(3-30 \mathrm{MHz})$, very high $(30-300 \mathrm{MHz})$ and even ultra-high (300MHz-3GHz) frequency systems (Leach, 2001). These efforts gear up tuning into a host of devices which make a promising alternative to traditional electronic components in the design of multiple RF devices (e.g. mixers, tuneable capacitors, inductors, switches, oscillators). The paper is organized as follows. Section II presents the design theory of MEMS Beam Resonator, two stage operational amplifier, oscillator including equation and circuit diagram. Section III provides simulations results of two stage amplifier.

\section{A. Mems Beam Resonator}

\section{Design Theory}

Fig. 1 shows the MEMS beam resonator structure. The polysilicon layers are used to define the MEMS resonator and electrodes. Here we have two polysilicon levels.

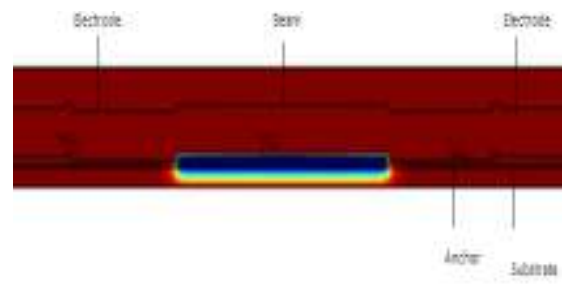

Fig. 1: MEMS Beam Resonator 
One level was used for the resonator while the other polysilicon level was used for electrodes. When AC voltage is applied to the electrode, DC voltage is applied to the anchor and an, producing the microstructure being driven into resonance. The resonators have a setup configuration with separate excitation electrode and output electrodes. The beam is hanged so that it can vibrate freely under the influence electrode applied under given DC condition. The applied AC voltage results in an electrostatic force between the electrode and the beam that results into consequently vibration of the resonator. In this case, the silicon dioxide layer between the two polysilicon levels defines the lateral gap between the electrode and beam resonator, allowing gaps as narrow as 0.5 um to be realized. Polysilicon thickness, which is typically a few 1 um and therefore much thinner than the polysilicon resonator and its resonance frequency can be defined as,

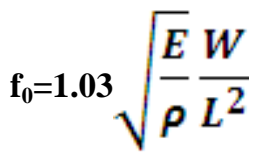

Where,

E: Young's modulus of the material

$\boldsymbol{\rho}$ : Mass density of the material

W: Beam width

L: Beam length.

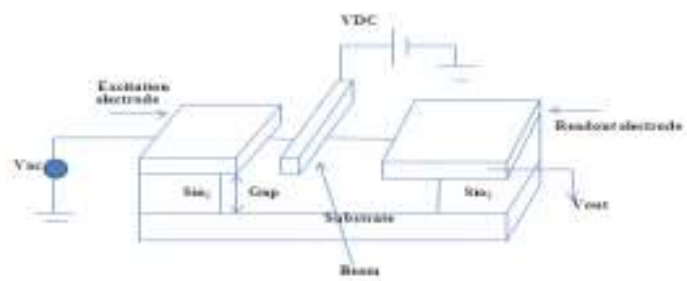

Fig. 2: Electrical simulation setup for MEMS Beam Resonator

According to the equation for resonance frequency the frequency increases as the length decreases. This oscillation of the beam changes the gap between the electrodes also changing the capacitance between the two electrodes. The variation in capacitance is maximum at $f 0$ and hence the current. Practically a mechanical transducer in the form of $\lambda / 4$ length is used to connect two such beams, the ac input signal is applied to moving electrode of first beam and the output current is measured at the moving electrode of the second beam. Hence, they are resulting in an electrostatic force between the electrode and the beam that consequently causes the vibration of the resonator. The capacitance change between the sense electrode and the beam due to the movement of the beam generates a current in the sense electrode. Table 1 shows all the design parameters of Beam resonator in this work. The natural oscillation frequency depends on the shape, physical dimension of the device, the mechanical properties of the material and the vibration mode.

Table 1: Design Parameter of MEMS Resonator

\begin{tabular}{|l|l|l|}
\hline Input Parameter & Value & Unit \\
\hline Beam length, $\mathrm{L}$ & 14 & $\mathrm{um}$ \\
\hline Beam width, W & 1 & $\mathrm{um}$ \\
\hline Electrode width, $\mathrm{W}_{\mathrm{e}}$ & 0.5 & $\mathrm{um}$ \\
\hline Beam thickness, $\mathrm{h}$ & 1 & $\mathrm{um}$ \\
\hline Electrode to resonator gap & \multicolumn{1}{|c|}{0.5} & $\mathrm{um}$ \\
\hline Poisson Ratio, $\mathrm{v}$ & 0.36 & -- \\
\hline Young Modulus, E & 64 & $\mathrm{GPa}$ \\
\hline Density of poly silicon, $\boldsymbol{\rho}$ & 2700 & $\mathrm{Kg} / \mathrm{m}^{3}$ \\
\hline
\end{tabular}

\section{B. Equivalent Circuit Model}

We make a few qualitative observations. First, the beam ends move in the opposite direction and the beam is acnored from the middle which is the vibrational nodal point. Secondly, the beam is actuated symmetrically using both electrodes and is listened from the center anchor making the resonator a two port device. Applying voltage over the resonator results in ac-current flowing through the device. This current can be divided into two parts: "normal" current $i_{a c}$ due capacitive current path between beam and electrodes and motional current imot due to motion of the beam end. 


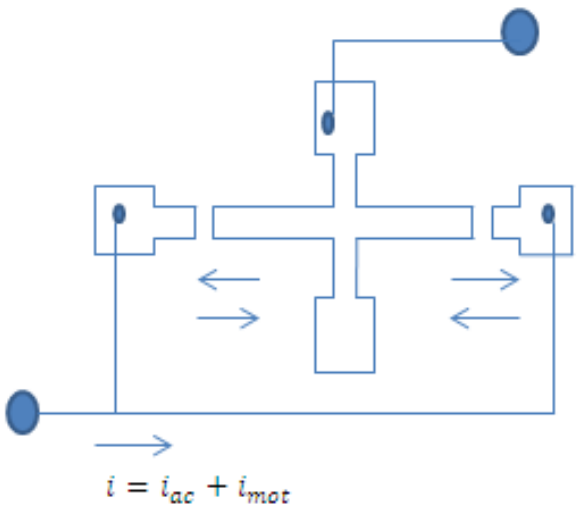

Fig. 3: Schematic of Beam Resonator

\section{Capacitive Excitation}

We use electrostatic force to actuate the beam. This requires a large dc voltage $\mathrm{v}_{\mathrm{dc}}$ over the narrow gap between the beam end and the electrode superposed by an ac voltage $\mathrm{v}(\mathrm{t})$ at the actuation frequency. The energy stored in the capacitor $\mathrm{C}$ formed by the electrode and the beam end.

$E=\frac{1}{2} C V^{2}$

$E=\frac{1}{2} C\left(v^{2} d c+2 v a c v d c+v^{2} \omega c\right)$

Thus, we have force at three frequencies: a dc-force, force at excitation frequency $f$ due to cross term $2 v_{a c} v_{d c}$, and force at twice the excitation frequency due to square term $\mathrm{v}^{2}$. The last term is very small so ignore it. The plate capacitance is

$c=\epsilon \frac{A e l}{d-x}$

Where, e is the permittivity of free space, Ael is the electrode area, $d$ is the initial electrode gap, and $x$ is again the movement of the beam tip. The force is obtained from

$f=\frac{\partial E}{\partial g}=v^{2} \epsilon \frac{A e I}{2(d-x)^{2}}$
$f=v_{a g} v_{d e} \epsilon \frac{A e l}{d^{2}}=\eta v_{a c}$

A

Where we have defined a new variable, the electro mechanical transduction factor,

$\eta=v_{d e} \frac{A e l}{d^{2}}$

\section{Resonator Current}

The current through the resonator is

$i=\frac{\partial C_{W}}{\partial t}=C \frac{\partial v}{\partial t}+v \frac{\partial C}{\partial t}$

With the approximations we take the equation,

$i=C_{0} \frac{\partial v}{\partial t}+n \frac{\partial x}{\partial t}$

\section{Electrical Equivalent Circuit}

We are now ready to develop an equivalent circuit for our beam resonator using above equations,

$\frac{M}{m} \frac{\partial i_{m}}{\partial t}+\frac{F_{i}}{\pi} i_{m}+\frac{k}{\pi} \int i_{m} d t=f(t)$

Next, remembering that $f=\square u a c$ 
$\frac{M}{\eta^{2}} \frac{\partial i_{m}}{\partial t}+\frac{Y}{\eta^{2}} i_{m}+\frac{k}{n^{2}} \int i_{m} d t=v_{a c}$

By defining motional resistance, motional capacitance, and motional inductance as,

$$
\begin{aligned}
& R_{\mathrm{m}}=\frac{\sqrt{k m}}{Q n^{2}} \\
& C_{\mathrm{m}}=\frac{n^{2}}{k} \\
& \mathbb{L}_{\mathrm{m}}=\frac{M}{n^{2}}
\end{aligned}
$$

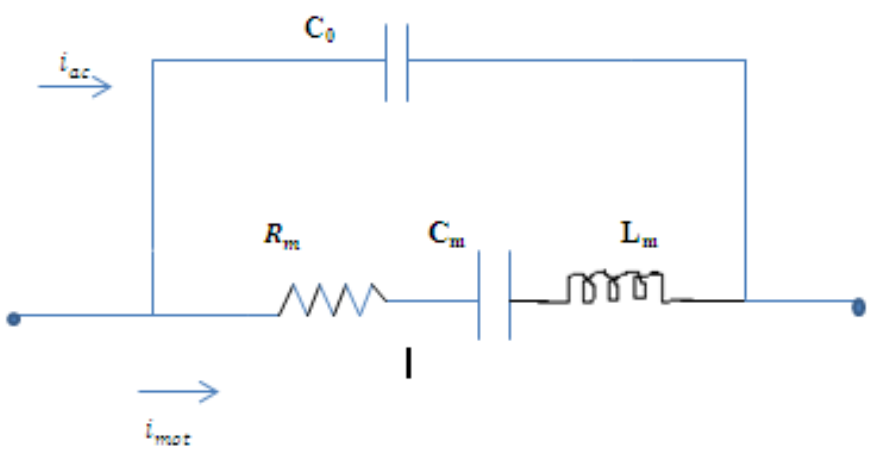

Fig. 4: Equivalent circuit of MEMS Resonator

\section{Amplifier Design}

Amplification is an essential function in most analog circuit. The block diagram of two stage Op-Amp. It consists of two stages in which first is differential stage and second is the gain stage. Output buffer is also used. In CMOS Op-Amp compensation network is used to which a feedback network is applied around the amplifier in virtually all Op-Amp applications. Transistors M1, M2, M3, and M4 form the first stage of the op amp the differential amplifier with differential to single ended transformation. Transistors M1 and M2 are standard N channel MOSFET transistors which form the basic input stage of the amplifier. The gate of M1 is the inverting input and the gate of M2 is the non-inverting input. A differential input signal applied across the two input terminals will be amplified according to the gain of the differential stage. The second stage is a current sink load inverter.

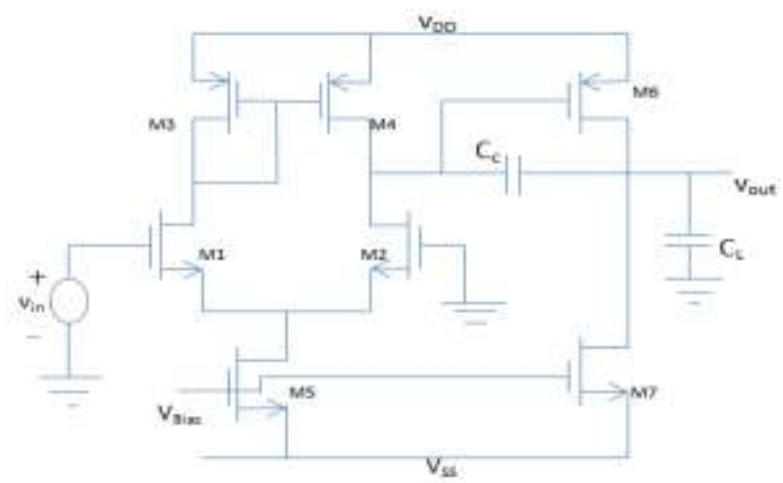

Fig. 5: Two Stage Operational Amplifier

The purpose of the second gain stage, as the name implies, is to provide additional gain in the amplifier. Consisting of transistors M5 and M6, this stage takes the output from the drain of M2 and amplifies it through M5 which is in the standard common source configuration. Again, similar to the differential gain stage, this stage employs an active device, M6, to serve as the load resistance for M5. The gain of this stage is the transconductance of M5 times the effective load resistance comprised of the output resistances of M5 and M6. M6 is the driver while M7 acts as the load. 
Table 2: Requirement of Differential Amplifier

\begin{tabular}{|l|l|l|}
\hline Design Specification & Value & Unit \\
\hline VDD & 1.8 & $\mathrm{v}$ \\
\hline Gain & 100 & $\geq 60 \mathrm{db}$ \\
\hline Load Capacitance & 2 & $\mathrm{Pf}$ \\
\hline Input Common Mode Range(+) & 1.6 & $\mathrm{v}$ \\
\hline Input Common Mode Range(-) & 0.8 & $\mathrm{v}$ \\
\hline Slew Rate & 20 & $\mathrm{v} / \mathrm{u} \mathrm{sec}$ \\
\hline Power Dissipation & $\leq 300$ & $\mathrm{uw}$ \\
\hline Gain Bandwidth & 30 & $\mathrm{MHz}$ \\
\hline Technology Used & 180 & $\mathrm{~nm}$ \\
\hline
\end{tabular}

\section{Design Procedure:}

- Find I5 from slew rate.

- M3, M4 get from $\operatorname{ICMR}(+)$

- M1, M2 get from Gain bandwidth.

- M5 get from ICMR(-)

- M8 will get from slew rate and M5.

\section{Slew rate}

Before finding slew rate we have to know, the value of $\mathrm{Cc}$.

$\mathrm{C}_{\mathrm{c}} \geq 0.22 \mathrm{CL}$

$\mathrm{C}_{\mathrm{L}}=2 \mathrm{PF}$

$\mathrm{C}_{\mathrm{c}} \geq 0.44 \mathrm{fF}$

Slew Rate $=\frac{I_{5}}{C_{e}}$

$\mathrm{I}_{5}=16 \mathrm{uA}$

Fixing ...I5=20Ua.

\section{Design of $M_{1}$ and $M_{2}$}

$$
\begin{aligned}
& \left(\frac{W}{L}\right)_{1,2}=\frac{g_{m 2}}{u_{n} c_{0 x}} \cdot 2 I_{d} \\
& \left(\frac{W}{L}\right)_{1,2}=\frac{g_{m 1}^{2}}{u_{n} c_{0 x}} \cdot I_{5} \\
& \left(\frac{W}{L}\right)_{1,2}=\frac{(160)^{2}}{300} \cdot 20 \\
& \left(\frac{W}{L}\right)_{1,2}=5
\end{aligned}
$$

Similarly we calculated width and length ratio for $\mathrm{m} 3, \mathrm{~m} 4, \mathrm{~m} 5, \mathrm{~m} 6, \mathrm{~m} 7, \mathrm{~m} 8$.

\section{Oscillator Design}

An Oscillator is basically an amplifier with "Positive Feedback", or regenerative feedback (in-phase) and one of the many problems in electronic circuit design is stopping amplifiers from oscillating while trying to get oscillators to oscillate. Oscillators work because they overcome the losses of their feedback resonator circuit either in the form of a capacitor, inductor or both in the same circuit by applying DC energy at the required frequency into this resonator circuit. In other words, an oscillator is an amplifier which uses positive feedback that generates an output frequency without the use of an input signal. It is self-sustaining. Then an oscillator has a small signal feedback amplifier with an open-loop gain equal to or slightly greater than one for oscillations to start but to continue oscillations the average loop gain must return to unity. In addition to these reactive components, an amplifying device such as an Operational Amplifier or Bipolar Transistor is required. Unlike an amplifier there is no external AC input required to cause the Oscillator to work as the DC supply energy is converted by the oscillator into $\mathrm{AC}$ energy at the required frequency.

$$
\begin{aligned}
& A_{v}\left(V_{\text {in }}-\beta V_{\text {out }}\right)=V_{\text {out }} \\
& A_{v} V_{\text {in }}-A_{v} \beta V_{\text {out }}=V_{\text {out }}
\end{aligned}
$$




$$
\frac{V_{\text {out }}}{V_{\text {in }}}=G v=\frac{1}{1+A \beta}
$$

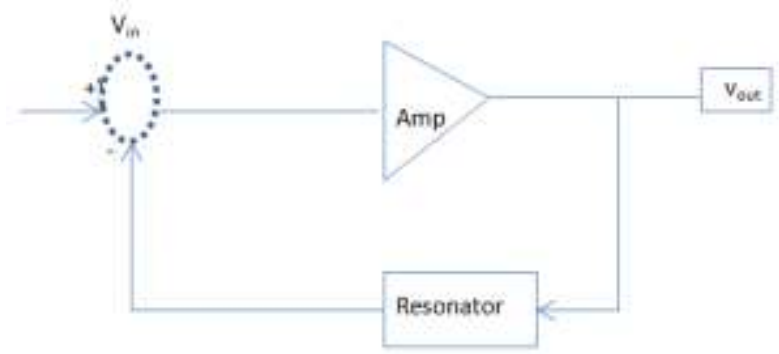

Fig. 6: Basic Feedback Oscillator Circuit

Oscillators are circuits that generate a continuous voltage output waveform at a required frequency with the values of the inductors, capacitors or resistors forming a frequency selective RLC resonant tank circuit and feedback network. This feedback network is an attenuation network which has a gain of less than one $(\beta<1)$ and starts oscillations when $A \beta>1$ which returns to unity $(A \beta=1)$ once oscillations commence.

\section{Simulation Result}

The two- stage CMOS Operational amplifier and MEMS Oscillator is done using Cadence software. Design parameters of two stage CMOS Op-amp and MEMS Resonator are summarized in table 3.

Table 3: Design Parameters

\begin{tabular}{|c|c|c|}
\hline TWO Stage Op-amp & & \\
\hline Transistor Name & Width & Length \\
\hline $\mathrm{M}_{1}, \mathrm{M}_{2}$ & 3 um & $500 \mathrm{~nm}$ \\
\hline $\mathrm{M}_{3}, \mathrm{M}_{4}$ & $7 \quad$ um & $500 \mathrm{~nm}$ \\
\hline $\mathrm{M}_{5}$ & $6 \quad$ um & $500 \mathrm{~nm}$ \\
\hline $\mathrm{M}_{6}$ & $87 \mathrm{um}$ & $500 \mathrm{~nm}$ \\
\hline $\mathrm{M}_{7}$ & $37.5 \mathrm{um}$ & $500 \mathrm{~nm}$ \\
\hline \multicolumn{3}{|l|}{ MEMS Resonator } \\
\hline Input parameter & Value & Unit \\
\hline Resistance, $\mathrm{R}_{\mathrm{M}}$ & 2.8 & $\mathrm{M} \Omega$ \\
\hline Inductance, $\mathrm{L}_{\mathrm{M}}$ & 13.6 & $\mathrm{H}$ \\
\hline Capacitance, $\mathrm{C}_{\mathrm{M}}$ & 2.46 & $\mathrm{aF}$ \\
\hline Feed through capacitance & 2 & $\mathrm{fF}$ \\
\hline
\end{tabular}

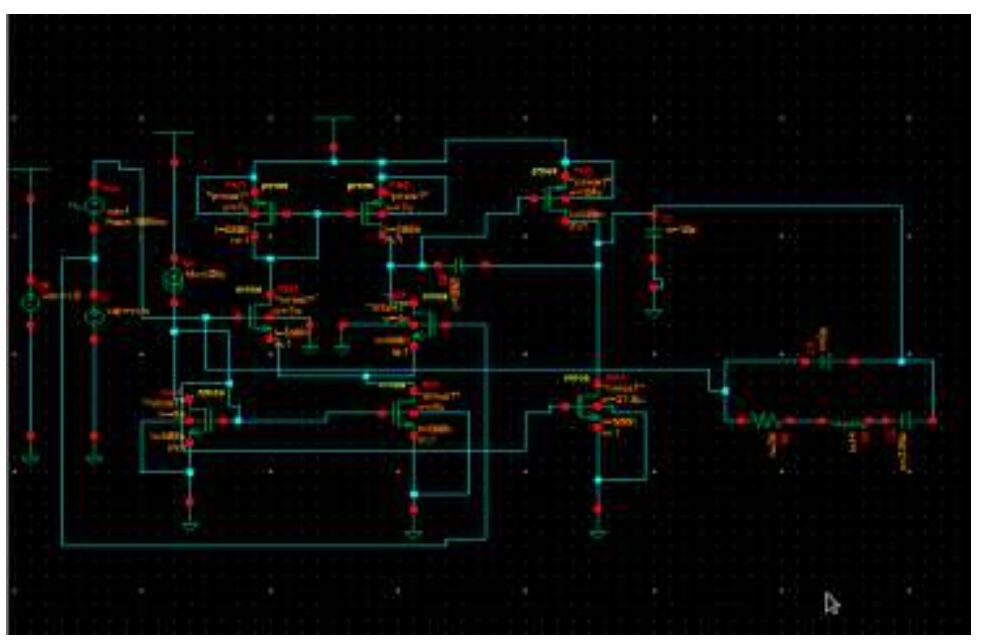

Fig. 7: Simulation circuit of MEMS Oscillator

Fig. 5 shows the designed circuit of two stage operational amplifier. The circuit is designed with Vss of $-1.8 \mathrm{v}$ and Vdd of 1.8v. Fig. 7 shows the proper simulated design of MEMS oscillator circuit with two stage operational amplifier. The circuit is designed with motional resistance of $R_{m}=2.8 \mathrm{M} \Omega$, capacitance, $C_{m}=2.46 \mathrm{aF}$ and inductance, $\mathrm{L}_{\mathrm{m}}=13.6 \mathrm{H}$ respectively. Two stage operational amplifier output is directly connected to input of equivalent circuit model of MEMS beam resonator. 


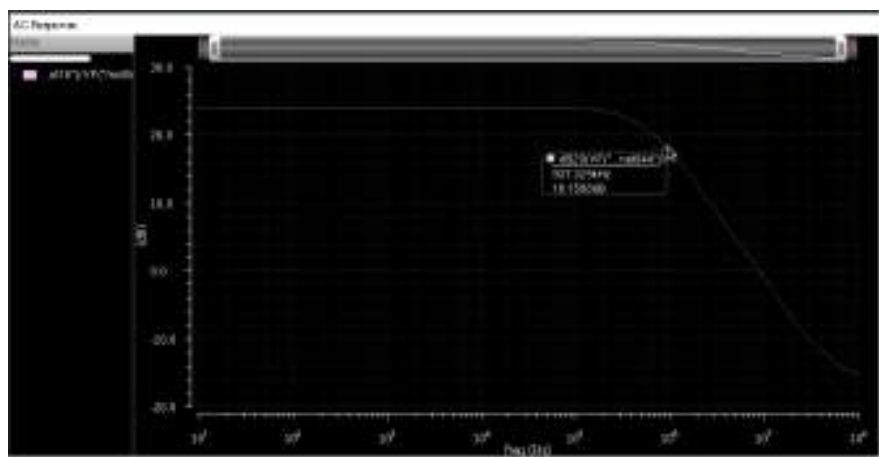

Fig. 8(A): AC Response of MEMS based Oscillator

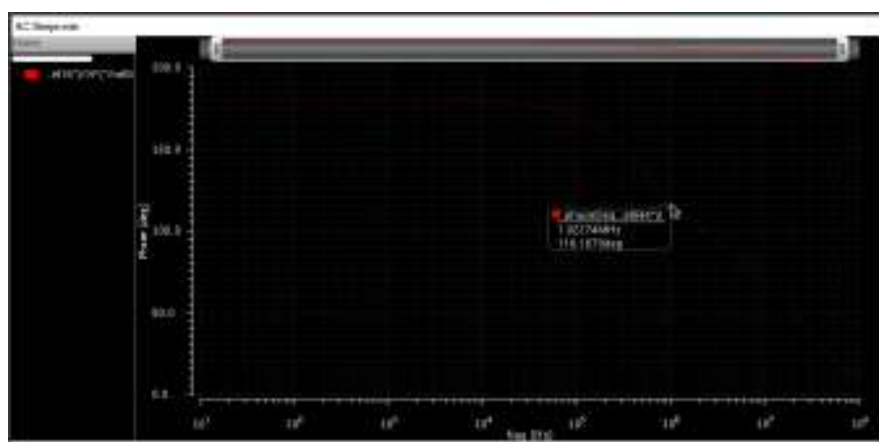

Fig. 8(B): AC Response of MEMS based Oscillator

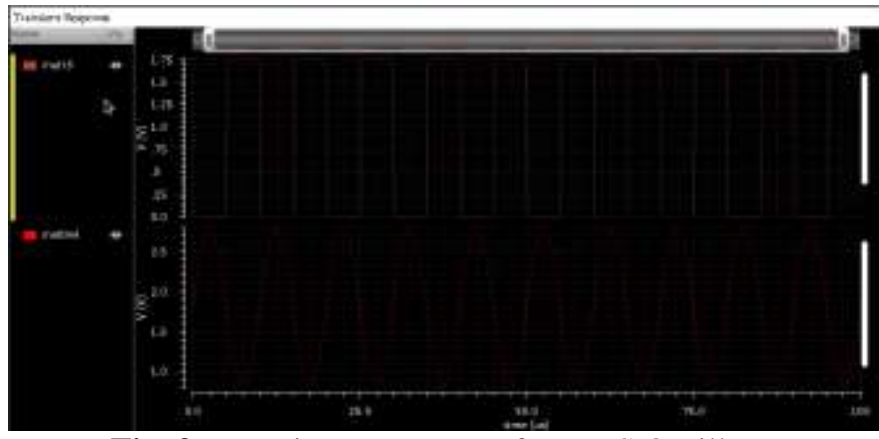

Fig. 9: Transient Response of MEMS Oscillator

Table 3 shows the design parameter of both two stage operational amplifier and MEMS Beam Resonator.

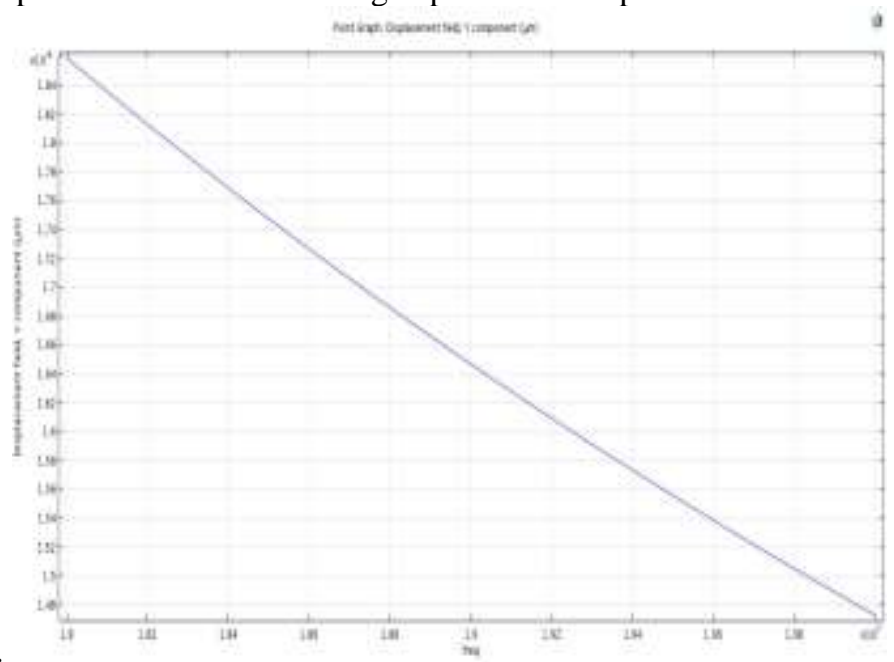

Fig. 10: Frequency Response of MEMS Oscillator 
Fig $8(\mathrm{a}$ and $\mathrm{b}$ ) provides $\mathrm{AC}$ response of the circuit which obtained by applying ac signal to the input of amplifier and measuring from output of MEMS Oscillator. A gain of $18.17 \mathrm{~dB}$ is obtained at $19.8 \mathrm{MHz}$ resonance frequency and also phase of the oscillator is 115 degree thus it satisfies the condition. Fig 9 provides transient response of MEMS oscillator providing the period of output signal is 0.1us. As depicted at Fig 10 shows the oscillation frequency is $19.8 \mathrm{MHz}$ obtained from frequency response of the MEMS beam resonator.

\section{Conclusion}

The MEMS beam resonator and two stage Operational amplifiers were designed and simulated. This methodology was able to achieve the $19.8 \mathrm{MHz}$ oscillation at gain $18.17 \mathrm{~dB}$ using $1.8 \mathrm{~V}$, VDD and phase of 115 degree at $19.8 \mathrm{MHz}$ compared to $13 \mathrm{~dB}$ in [1]. Further Improvement shall be done for this CMOS-MEMS oscillator in term of its oscillation frequency and phase noise performance.

\section{Acknowledgement}

The authors would like to acknowledge the support provided by the centre in VLSI Lab, SRM University.

\section{References}

[1]. Syamsi Taufik, S.Khan, A.N.Nordin , 2014, “ Design and Simulation of MEMS Oscillator” Proc. IEEE 978-1-4673-6386-0/14/..

[2]. Leach, R., Cui, Z., and Flack, D (Eds) , 2001: 'Microsystems technology standardization roadmap'. 'Project IST-2001-37682 funded by the EUIST program.

[3]. W.T.Hsu, "Vibrating RF MEMS for Timing and Frequency References", 2006 IEEE MTT-S International Microwave Symposium, pp. 672-675.K. Elissa, "Title of paper if known," unpublished.

[4]. R. Nicole, "Title of paper with only first word capitalized," J. Name Stand. Abbrev., in press.

[5]. Y. Yorozu, M. Hirano, K. Oka, and Y. Tagawa, "Electron spectroscopy studies on magneto-optical media and plastic substrate interface," IEEE Transl. J. Magn. Japan, vol. 2, pp. 740-741, August 1987 [Digests 9th Annual Conf. Magnetics Japan, p. 301, 1982].

[6]. M. Young, The Technical Writer's Handbook. Mill Valley, CA: University Science, 1989.

[7]. B.Razavi, [2001] “Design of Analog CMOS Integrated Circuits,” New York:McGraw-Hill,pp483-484.

[8]. R.Cerda, "Pierce-Gate Crustal Oscillator, an Introduction", Crytek Corporation,pp.12.

[9]. J.Karim, A.Nordin, and Alam, A.H.M Zahirul 2012. "Design of a pierce oscillator for CMOS Saw Resonator, International Conference on computer and Communication Engineering (ICCCE 2012), Kuala Lumpur"

[10]. F.Nabki, Allidina.K, Ahmad.F, 2009. "A high gain-bandwidth product transimpedance amplifier for MEMS -based oscillator'.pp.454. 